denn?) den Westen zur „Umkehr“ zu bewegen! Kann man übersehen, daß das oft als moskau-abhängig gescholtene Guinea-Bissao bereits EG-assoziert ist (wann folgen Mozambique und Angola?)? Ist der Westen in Afrika nicht viel zu präsent - auf Kosten afrikanischer Self-Reliance?

Dikussionswürdig ist schließlich auch die Grundannahme Anprengers, daß auch in der RSA den Afrikanern die "nationale Befreiung " gelingen wird (im Sinne der Majority rule!) - obwohl doch alle Anzeichen eher für eine Balkanisierung oder Teilung des Landes sprechen.

Gerade auch wegen seiner provokanten und versuchsweise zukunftantizipierenden Thesen - die sich bei Ansprenger auf der Grundlage großer Sachkenntnis aufbauen - ist dieses aktuelle Buch für alle, die besorgt die Entwicklung im südlichen Afrika verfolgen, von großem Nutzen und besonderem Reiz: man ist aufgefordert weiterzudenken.

\title{
LeOnhard Harding
}

\section{Afrikanische Politik im südlichen Afrika}

Studien zum Konflikt im südlichen Afrika. Entwicklung und Frieden. Wissenschaftliche Reihe 9.

München und Mainz 1975, 210 S.

In seinem zweiten Beitrag zu den „Studien“ analysiert Harding die Organisationen und strategischen Ziele der afrikanischen Akteure, die entweder als Kollaborateure oder als radikale Verweigerer der weißen Minderheit in der RSA gegenüberstehen. Dabei werden interessante Informationen über die "Strategie der taktischen Anpassung " seitens der Homeland-Führer Mantanzima, Mangope und Buthelezi gegeben sowie über die damit konfligierende "Strategie des Disengagements", die vor allem von der schwarzen Studentenorganisation SASO und der „Black Consciousness"-Bewegung unter den urbanisierten Weißen außerhalb der Bantustans vertreten wird. Dabei gelingt es Harding recht überzeugend nachzuweisen, daß die (zunächst als Ausgangshypothese postulierte, einzig mögliche) Strategie der Anpassung der Homeland-Leader an die Strukturen des von den Weißen erzwungenen "Separate development" das gew ünschte Ziel - den Systemwechsel — nicht erreichen könne, „aus politischen, vor allem aber aus wirtschaftlichen und noch stärker aus militärischen Gründen " (S. 75).

In der (beeindruckend dokumentierten) Black Consciousness-Bewegung in den Städten sieht Harding "Elemente einer prärevolutionären Situation“ verwirklicht, fügt aber hinzu, daß diese Strategie der Verweigerung (mit deutlichen Unterströmungen von Black Power und schwarzem Rassismus) „auf absehbare Zeit ohne klar definierte Erfolgschancen" sei (S. 98).

Auch die Nachbarstaaten - BLS-Länder, Malawi und Sambia - werden in die Betrachtung herrschaftsverändernder Prozesse und Perspektiven einbezogen, wobei der Autor z. B. bezüglich Sambias zu dem freilich (nicht gerade umwerfenden) Schluß gelangt: Im Interesse einer Konfliktlösungsstrategie ergäbe sich für die BRD als Ansatz einer „Interventionsmöglichkeit“, „Sambia bei seinem Bemühen um ein Disengagement vom Süden zu unterstützen, um auf diese Weise Pretorias Vordringen zum Norden zu stoppen und einen Machtwechsel in Rhodesien zu beschleunigen" (S. 132). Als wenn die RSA auf die Tomatenexporte nach Sambia angewiesen wäre! 
Im Schlußkapitel unterstreicht Harding die These, daß der „größte Gegensatz der gegenseitigen Strategien der zwischen der Regierung der Republik Südafrika und der der ,Urban Africans"“ sei (S. 133). Ebensowenig hätten aber die schwarzen kooperationsbereiten Bantustan-Politiker - von der OAU und der UNO nicht anerkannt, ohne gemeinsames Handlungskonzept und letztlich ohne Legitimationsbasis - eine reale Chance, einen Systemwechsel zu bewirken (möglich wären höchstens marginale Systemänderungen). Da keine einzelne Strategie Erfolg verspräche, müßte man für eine „multiple Strategie“ eintreten: „Erfolgsaussichten können der , afrikanischen Politik' nur dann eingeräumt werden, wenn ihre verschiedenen Ansätze, einschließlich der militärischen Aktivitäten der Befreiungsbewegungen, gleichzeitig und gemeinsam weiterverfolgt werden." (S. 135) Nur in eine solche "Globalstrategie“ könne die "Anpassungsstrategie der HomelandRegierungen" - die an sich allein systemstabilisierend wirke - sinnvoll eingebaut werden — „mit dem langfristigen Ziel eines Systemwechsels“.

Die praxeologischen Schlußfolgerungen Hardings aus seiner insgesamt sehr lesenswerten Fakten- und Trendanalyse sind wohl doch etwas zu pragmatisch! So kann z. B. der politisch-strategische Konflikt zwischen rivalisierenden schwarzen Gruppen in der RSA schwerlich dadurch ausgeräumt werden, daß westliche Industriestaaten nun alle schwarzen Gruppen - vom opportunistischen Kaiser Mantanzima bis zu bewaffneten Freiheitskämpfern - "gleichzeitig“ anerkennen und unterstützen. Zumindest sollte man von einer als Handlungsanweisung gedachten Konfliktlösungsstrategie erwarten dürfen, daß die empfohlenen Teilstrategien (ganz abgesehen von dem unterschiedlichen Grad ihrer jeweiligen Erfüllungswahrscheinlichkeit) auf ihre zu erwartenden Wirkungen befragt und in ihrer Relevanz gestaffelt werden. Warum schließlich wurden Fragen nach den Modalitäten (friedlich?) eines Systemwechsels (den Harding als in jeder Hinsicht wünschenswert bezeichnet) als Folge solcher Unterstützung von außen ausgeblendet? Was bedeutete denn eine Unterstützung der militanten „urban blacks“ in SOWETO durch sagen wir Gruppen aus EG-Staaten (als Teil der geforderten „multiplen Strategie“?).

Vielleicht sind solche Fragen ohne die Beschäftigung mit Theoremen über imperialistische und kapitalistische Herrschafts- und Abhängigkeitsstrukturen doch nicht zu beantworten!

Trotz der hier vorgebrachten Fragen, Zweifel und Einwände stellen die vier ersten Bände der „Studien zum Konflikt im südlichen Afrika“ einen konkret nützlichen und sehr wichtigen Diskussionsbeitrag zu einem hoch aktuellen Krisenherd der Dritten Welt dar - nützlich deshalb, weil hier ein bisher unübersichtliches Konfliktfeld mit seinen regionalen und überregionalen Dimensionen und Trends im wahrsten Sinne des Wortes aufgearbeitet worden ist (das bezeugen auch die umfangreichen Quellennachweise und die Bibliographien), und wichtig vor allem auch deshalb, weil die informationsreichen "Studien" gerade auch aufgrund ihrer argumentativen Direktheit, Kühnheit und nicht selten Vagheit dazu geeignet erscheinen, die Südafrikadebatte in der Bundesrepublik zu stimulieren und zu strukturieren. $\mathrm{Ob}$ der Anspruch eingelöst werden kann, politikberatend und handlungsfördernd - im Sinne des kritischen Friedensbegriffs - zu wirken, darf dagegen bezweifelt werden. Es ist zu hoffen, daß die noch folgenden Bände der „Studien“ in diesem Punkt mehr Klarheit bringen.

Rainer Tetzlaff 